\title{
FDG dose extravasations in PET/CT: frequency and impact on SUV measurements
}

\author{
Medhat M. Osman ${ }^{1,2}{ }^{*}$, Razi Muzaffar ${ }^{1}$, M. Erkan Altinyay ${ }^{1}$ and Cyrus Teymouri ${ }^{3}$ \\ 1 Division of Nuclear Medicine, Department of Radiology, Saint Louis University, St. Louis, MO, USA \\ ${ }^{2}$ Saint Louis VA Medical Center, St. Louis, MO, USA \\ ${ }^{3}$ Nuclear Medicine Technology Program, Medical Imaging and Radiation Therapeutics, Doisy College of Health Sciences, Saint Louis University, St. Louis, MO, USA
}

Edited by:

Aradhana M. Venkatesan, NIH Clinical Center, USA

\section{Reviewed by:}

Jonathan McConathy, Washington

University in St. Louis, USA

Esma Akin, The George Washington

University Hospital, USA

\section{*Correspondence:}

Medhat M. Osman, Division of Nuclear Medicine, Department of Radiology, Saint Louis University, 3635 Vista Avenue, 2-DT, St. Louis, MO 63110, USA.

e-mail:mosman@slu.edu

\begin{abstract}
Objectives: Positron emission tomography (PET)/CT with 18F-FDG has proven to be effective in detecting and assessing various types of cancers. However, due to cancer and/or its therapy, intravenous (IV) FDG injection may be problematic resulting in dose extravasations. In the most frequently used field of view (FOV), arms-up, and base of skull to upper thigh [limited whole body (LWB)], the injection site may not be routinely imaged. The purpose of this study was to evaluate the frequency of dose extravasations in FDG PET and the potential impact on standard uptake value (SUV) measurements. Methods: True whole body FDG PET/CT scans (including all extremities) of 400 patients were retrospectively reviewed. A log recorded cases of IV dose extravasations. When possible, SUVs were measured in two frequently used reference locations: mediastinum and liver. The SUVS were obtained in the same patients who had studies with and without FDG extravasations within an average of 3 months without interval therapy. Results: Of the 400 scans, 42 $(10.5 \%)$ had extravasations on the maximum intensity projections images. In scans with or without dose infiltration, FDG injection site was at or distal to the antecubital fossa in $97 \%$ of studies. Of those 42 cases, dose infiltration was within the LWB FOV in 29/42 (69\%) and outside in the remaining 13/42 (31\%). Of those 42 patients, 5 had repeat PET studies with no interval therapy. For those 5 patients, liver maximum SUV was $11.7 \%$ less in patients with infiltration than those without ( $2.22 \pm 0.54$ vs. $2.48 \pm 0.6)$. Mediastinum SUVmax was $9.3 \%$ less in patients with infiltration than those without $(1.72 \pm 0.54$ vs. $1.88 \pm 0.49)$. Conclusion: We conclude dose extravasations were commonly encountered $(10.5 \%)$ in PET/CT. However, it is underreported by at least $31 \%$ due to omitting injection site from the FOV. When present, extravasations may lead to underestimation of SUVmax. Therefore, it should not only be avoided but also reported in order to avoid false interpretations of the exam.
\end{abstract}

Keywords: FDG dose extravasations, whole body PET, PET/CT, FOV

\section{INTRODUCTION}

Positron emission tomography (PET) using fluorine-18-2-deoxyD-glucose $\left({ }^{18} \mathrm{~F}-\mathrm{FDG}\right)$ diagnoses, stages, and restages many cancers with an accuracy ranging from 80 to $90 \%$ and is often better than anatomical imaging (Czernin et al., 2007). Since the introduction of PET/CT, numerous studies showed that this whole body (WB) dual-modality imaging is better than PET or CT alone for staging and restaging most cancers (Czernin et al., 2007). Consequently, by 2006, the major vendors no longer offered PETonly systems, and by 2010, over 5000 combined PET/CT systems were in clinical operation worldwide (Mawlawi and Townsend, 2009; International Survey of PET/CT Operations and Oncology Imaging, 2010). The improvement in accuracy coupled with the convenience of presenting anatomical and functional information to physicians has rendered PET/CT imaging as the most important cancer imaging modality at the present time (Bar-Shalom et al., 2003). Furthermore, the use of PET/CT has been advocated as a first-line imaging modality for WB tumor staging, restaging, and assessing response to therapy in different types of neoplasm (Antoch et al., 2003).

In oncology, WB PET/CT is typically performed from the head to the pelvic floor (Von Schulthess et al., 2006). The use of the term WB is misleading since the most commonly used field of view (FOV) for the arms-up PET/CT WB protocol only includes the base of skull to upper thighs, thus limited WB (LWB), and does not include the brain, skull, and significant portions of both upper and lower extremities. In addition, the arms of patients undergoing PET/CT for cancer evaluation should be extended over the head except in head and neck cancers in which arms should be positioned along the sides (Delbeke et al., 2006). Therefore, the most commonly used injection site, the antecubital fossa, is frequently not included in the imaged FOV. Furthermore, due to cancer and/or its therapy, intravenous (IV) FDG injection may be problematic and occasionally result in dose extravasations. In a previous clinical study from this same site, true whole body (TWB) image acquisition, from the top of the skull to the bottom of the 
feet, revealed the presence of new, previously unidentified malignant sites outside the typical LWB FOV in $4 \%$ of patients (Osman et al., 2010). Consequently, we use TWB as the standard of care in all our cancer patients. In so doing, the injection site, with or without dose infiltration, is always included in the imaged FOV. The objective of the current study was to evaluate the frequency and pattern of dose extravasations in FDG PET and, when possible, to investigate potential impact of such extravasations on standard uptake value (SUV) measurements.

\section{MATERIALS AND METHODS PATIENTS}

A total of 400 consecutive PET/CT scans for patients referred for clinical evaluation of known or suspected malignancy between May 2006 and October 2006 were retrospectively evaluated for the presence of FDG extravasations at the injection site. All IV injections were performed by three experienced technologists (Certified Nuclear Medicine Technologist and PET certified) injecting $>1800$ cases/year. This retrospective study was approved by the Institutional Review Board and the need for informed consent was waived.

\section{PET INJECTION TECHNIOUE}

The standard F-18 FDG intravenous injection in our facility was performed using a 23 or 25 gage butterfly needle. The needle was inserted into a vein and connected to a three-way stopcock. The stopcock had saline and an empty $3 \mathrm{ml}$ syringe attached. After placing the needle into the vein, the technologist drew blood into the empty $3 \mathrm{ml}$ syringe. This blood was used to check the patient's blood glucose level. The technologist then flushed the line with the saline attached to the stopcock. After the blood glucose level was checked and within the acceptable range, the technologist measured the FDG activity in the dose calibrator and brought the dose into the injection room. The technologist unscrewed the 3$\mathrm{ml}$ blood syringe from the stopcock and attached the FDG dose. The activity was injected through the butterfly needle and flushed with 10-20 ml saline. After FGD injection and saline flush, the butterfly needle was removed from the patient. The injection site was covered with gauze and tape to stop bleeding at the site. The technologist then performed a post-injection measure of the dose syringe to calculate the net injected dose.

\section{PET/CT SCANNING}

Patients fasted at least $4 \mathrm{~h}$ before the PET acquisition and received an intravenous injection of approximately $5.18 \mathrm{MBq} / \mathrm{Kg}$ $(0.14 \mathrm{mCi} / \mathrm{Kg})$ of ${ }^{18} \mathrm{~F}-\mathrm{FDG}$, with a maximum of $444 \mathrm{MBq}$ $(12 \mathrm{mCi})$. Blood glucose level was measured immediately prior to FDG injection and was $<200 \mathrm{mg} / \mathrm{dl}$ in all patients. Patients were instructed to sit in a quiet injection room without talking during the subsequent $60 \mathrm{~min}$ of the FDG uptake phase and were allowed to breathe normally during image acquisition without specific instructions. All scans were acquired using a PET/CT scanner (Gemini; Philips Medical Systems), with an axial co-scan range of $193 \mathrm{~cm}$.

\section{CT SCANNING}

The CT scan component of the PET/CT scanner consisted of a 16 slice multidetector helical CT. Gantry allows for a patient port of
$70 \mathrm{~cm}$. Parameters were as follows for 12-13 bed acquisitions (from the top of the head through the bottom of the feet): $120-140 \mathrm{KV}$ and 33-100 mAs (based on body mass index), 0.5 s per CT rotation, pitch of 0.9 and $512 \times 512$ matrix. CT acquisition was performed before emission acquisition. CT data were used for image fusion and the generation of the CT transmission map. In all patients, the arms were placed above the patient's head for CT acquisition except in patients with head and neck cancers where the arms were placed at the patient's sides. The CT images were obtained without oral or IV contrast according to the usual PET/CT protocol in our institution.

\section{PET SCANNING AND IMAGE PROCESSING}

The PET component of the PET/CT scanner was composed of gadolinium oxyorthosilicate (GSO)-based crystals. Emission data were acquired for $12-13$ bed positions ( $193 \mathrm{~cm}$ coverage, identical to the CT protocol). Emission scans were acquired at $3 \mathrm{~min}$ per bed position. The FOV was from the top of the head to the bottom of the feet in all patients. The three-dimensional (3D) TWB acquisition parameters consisted of a $128 \times 128$ matrix and an $18-\mathrm{cm}$ FOV with a $50 \%$ overlap. Processing consisted of the $3 \mathrm{D}$ row action maximum likelihood algorithm (RAMLA) method (Browne and De Pierro, 1996). Total scan time per patient was 36-39 min.

\section{IMAGE ANALYSIS}

For each of the 400 patients included in this study, PET/CT reports were reviewed for the reported injection site. TWB static maximum intensity projection (MIP) PET images were retrospectively evaluated on Syntegra workstations (Philips Medical Systems), and a log was kept to record cases of FDG dose extravasations at the injection site and this was compared to the injection site documented in the PET/CT report. A dose was considered extravasated if any remaining FDG activity was visible at the site of injection within the body. In addition, a note was made of the location of the injection site. Dose extravasations cases were categorized into two groups; those within the routinely used base of skull to upper thigh (LWB) FOV and those with extravasations occurring outside the typical LWB, i.e., can only be detected by TWB image acquisition.

\section{SUV MEASUREMENTS}

Five patients had repeat PET studies within an average of 3 months without interval therapy for close follow-up. Each patient had a study both with and without FDG extravasation. In an attempt to evaluate potential impact of dose extravasations on quantitative measurements, maximum SUVs were measured $\left(\mathrm{ROI}=500 \mathrm{~mm}^{2}\right)$ in two frequently used anatomical reference locations: liver (mid right lobe) and mediastinum blood pool (aortic arch). For those cases, all available data was compared to select patients who had normal, almost similar, blood glucose levels in both studies and had no weight changes between studies. Image acquisition parameters were kept the same in all studies.

This retrospective study was approved by the Institutional Review Board and the need for informed consent was waived.

\section{RESULTS}

Of $400 \mathrm{PET} / \mathrm{CT}$ scans, FDG injection was performed at or distal to the antecubital fossa in $388(97 \%)$ of reviewed reports. Of those 
400 scans, $42(10.5 \%)$ had extravasations noticeable on the MIP images. Of those 42 cases, $29(69 \%)$ of the extravasations were noted within the LWB (Figure 1) whereas the remaining 13 (31\%) were outside the LWB imaged FOV (Figure 2). Of the 42 studies with dose extravasations, injection site included: the right antecubital fossa in 17 (40\%), the left antecubital fossa in 12 (29\%), the right wrist $7(17 \%)$, the left wrist in $3(7 \%)$, chest chemotherapy port was used for FDG injection in $2(5 \%)$ cases and injection was done in the right neck in a single case $(2 \%)$.

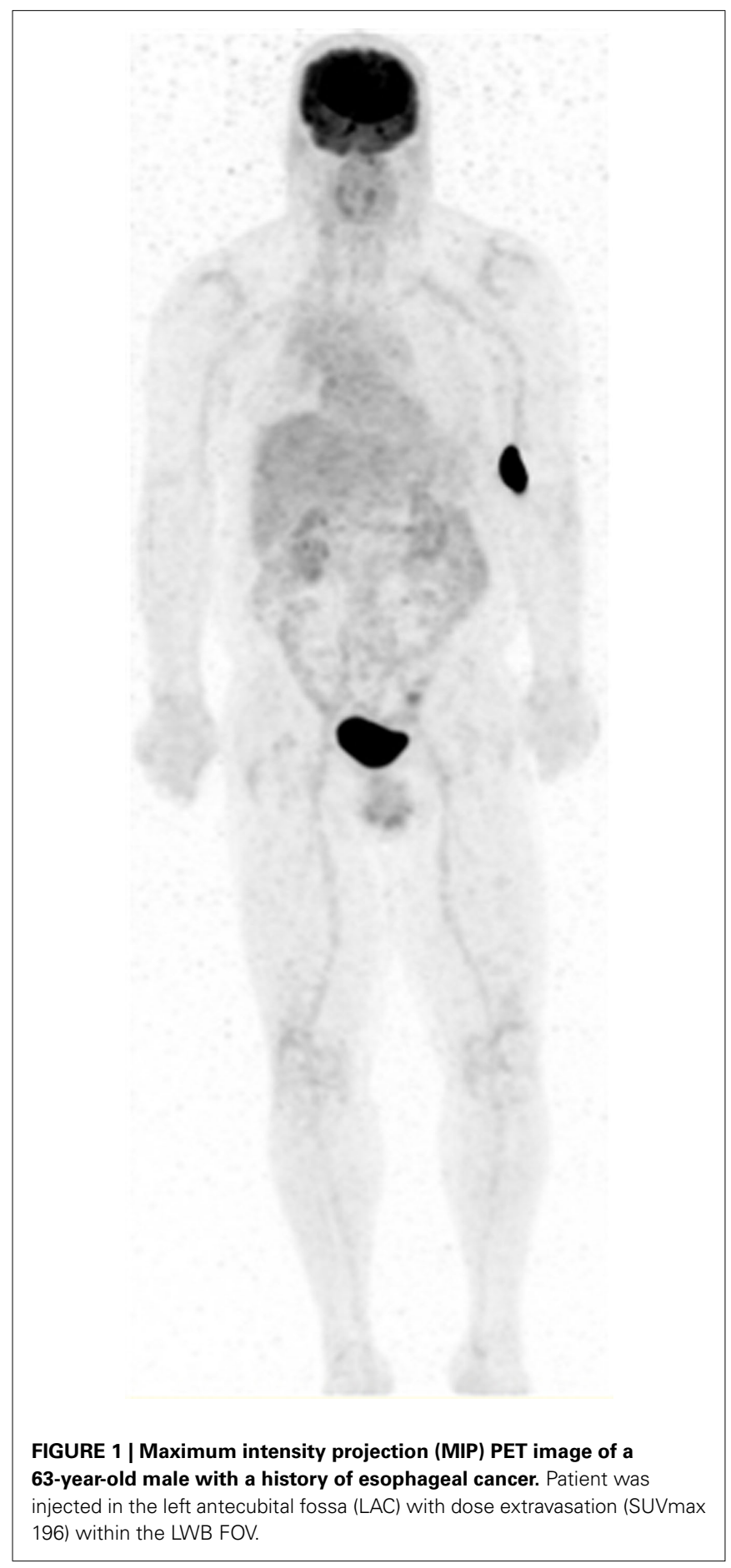

Of those 42 patients, 10 had repeat PET studies with and without tracer infiltration within the observation period. Of those 10, only 5 had no interval therapy with almost similar blood glucose level and no weight changes between the scans. Therefore, those 5 met inclusion criteria and were selected for ROI analysis and SUV measurements. Mean liver SUV max was $11.7 \%$ less in patients with infiltration than those without $(2.22 \pm 0.54$ vs. $2.48 \pm 0.6)$ and mean mediastinal SUV max was $9.3 \%$ less in patients with infiltration than those without $(1.72 \pm 0.54$ vs. $1.88 \pm 0.49)$. An example is presented in Figure 3.

\section{DISCUSSION}

The National Oncologic PET Registry (NOPR) evaluated 40,863 PET studies done at 1368 centers and concluded that FDG PET changed management for $38 \%$ of cases ( $95 \%$ confidence inter$\mathrm{val}=37.6-38.5 \%)$ across cancer types, providing strong evidence

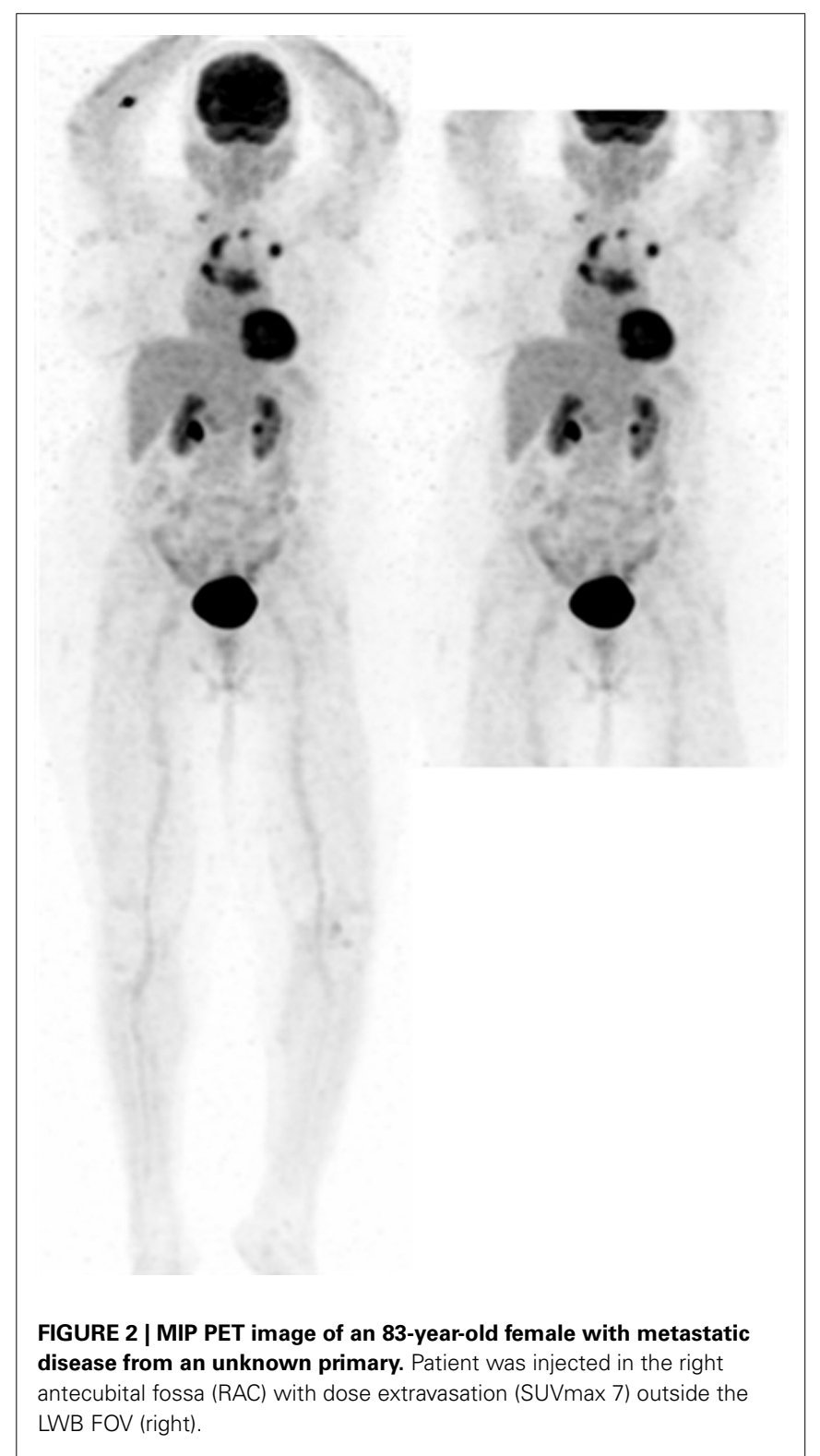




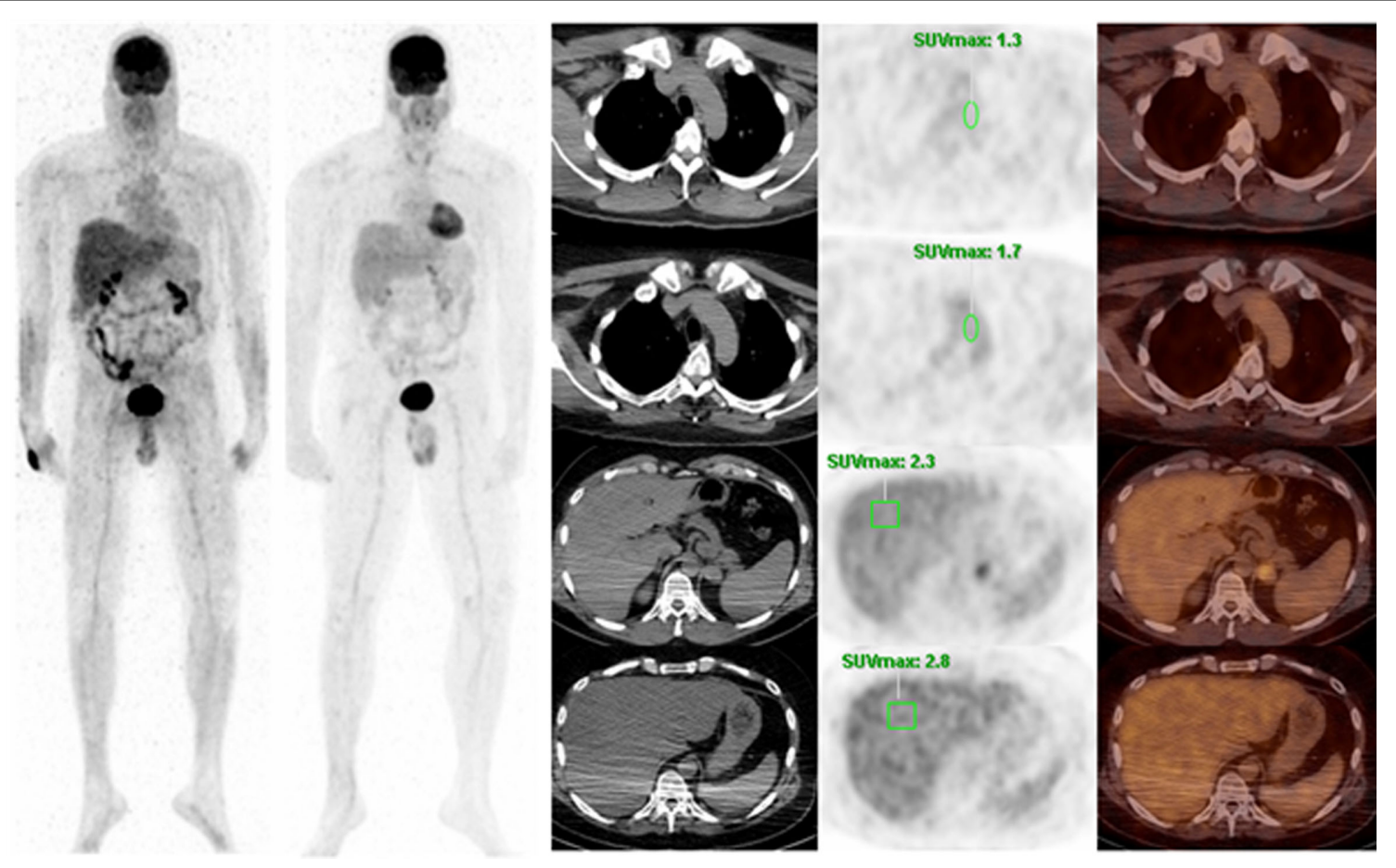

FIGURE 3 | Patient is a 46-year-old male with a history of melanoma. Left MIP shows extravasation in the right wrist and a repeat PET was performed 5 months later with no extravasation. The top row shows SUV measurement of the mediastinum with extravasation and the second row shows SUV of the mediastinum without extravasation. The third row shows SUV of the liver with extravasation and the bottom row shows SUV of the liver without extravasation.

that FDG PET is useful for many types of cancer and indications beyond those currently reimbursed by Centers for Medicade and Medicare Services (CMS; Hillner et al., 2008). In addition, FDG PET is currently plays a growingly critical role in assessing response to therapy in several tumor types. A relatively recent analysis of the NOPR data showed that 19\% of the registered scans were performed for treatment monitoring (Hilner et al., 2008). A recent follow-up publication concluded that essential elements that should be included in oncologic PET reports were missing. Therefore, many reports are less helpful to referring physicians, may lead to misdiagnosis, and may cause coding and billing errors (Coleman et al., 2010). According to the same study, FOV scanned and route of FDG administration were absent from the PET/CT reports evaluated by NOPR in 8.4 and $31 \%$, respectively. Further, this study did not evaluate location of injection site, in terms of being in or out the imaged FOV, the presence of FDG dose extravasations and potential impact of such extravasations on SUV measurements. Such data would be difficult to obtain in the most commonly used scanned FOV, base of skull to upper thigh, because the injection site is typically outside the FOV. In clinical practice, visual inspection of PET images is the main tool for image interpretation. However, the reading physician would not be able to incorporate the presence of dose infiltration and potential impact on the degree of metabolic activity if injection site was not included in the imaged FOV. In this study, $400 \mathrm{PET} / \mathrm{CT}$ scans were reviewed and extravasation was noted in 42 cases $(10.5 \%)$. Of the 42 cases with extravasations, dose infiltration was within the LWB
FOV in 29 (69\%) and outside in the remaining 13 (31\%). Since the antecubital fossa is the most common site of injection, many of such cases would be outside the LWB FOV except in head and neck cases where the arms are at the patient's side as the standard protocol.

Since 1993 when quantitative F-18 FDG PET was introduced for the early sequential monitoring of tumor response, there has been a growing interest in using FDG PET in assessing response to therapy (Wahl et al., 1993). Of the many different ways to monitor tumor response, SUV is the most widely applied and generally correlates well with more complex analytic approaches (Graham et al., 2000; Krak et al., 2003). To achieve reproducible SUV measurements, absolute and rigorous standardization of the protocol for PET image acquisition is required. On one hand, an SUV drop of more than $20 \%$ is considered a response on the basis of reproducibility considerations (Weber, 2005). According to the EORTC, $15 \%$ decline in SUV is considered response (Young et al., 1999). On the other hand, in a test-retest setting, the reproducibility of SUV measurements has varied. Boellards recently reported that variation in camera calibration, image reconstruction, and data analysis and/or settings can have more than a $50 \%$ effect on the measured SUV (Bollaard, 2009). The same publication reported that the effect of paravenous administration of FDG on SUV measurements is in the range of $0-50 \%$ or more, strongly depending on quality of administration. However, this was reported as estimated values based on unpublished data. 


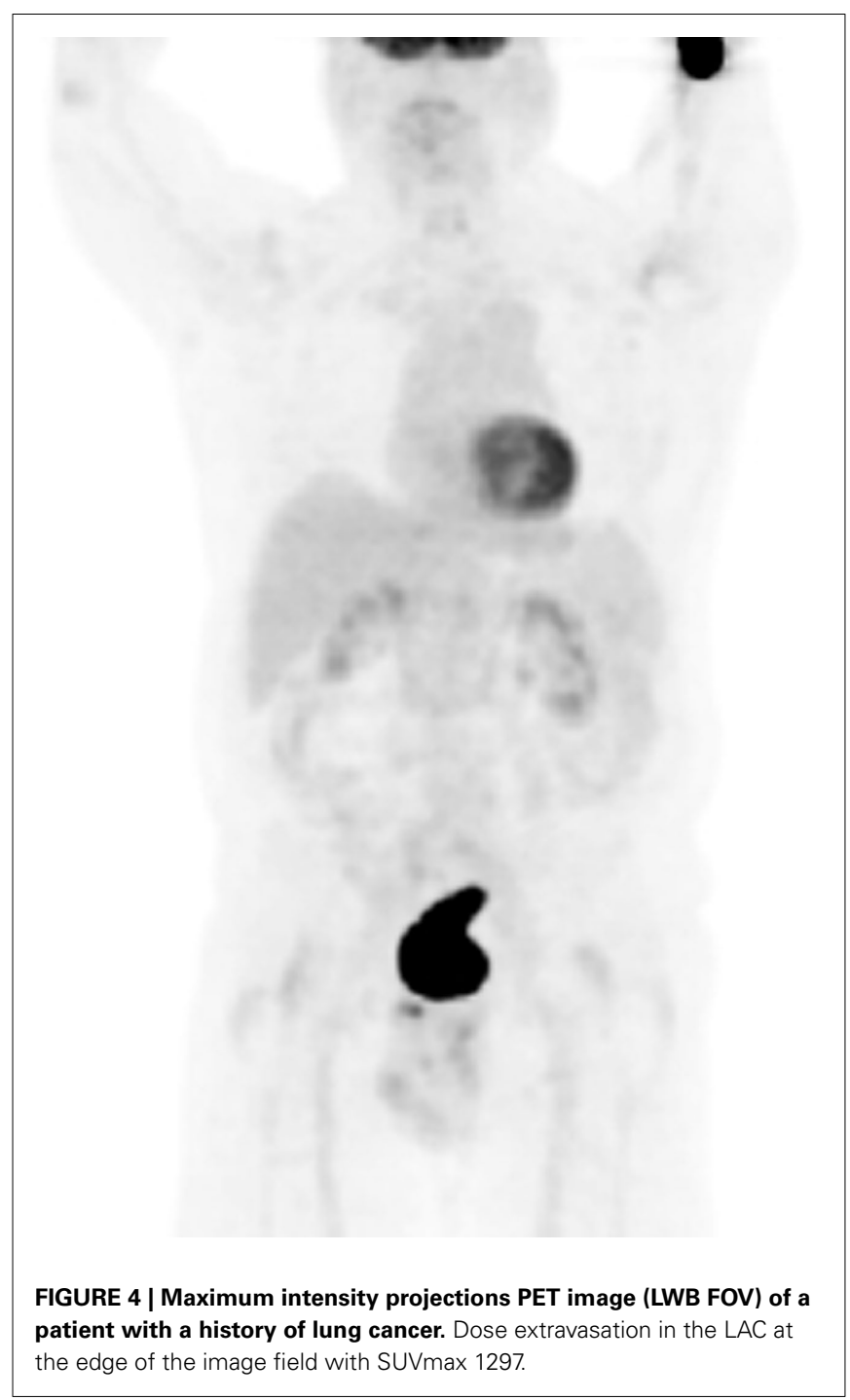

Comparing tumor activity to an internal reference point such as liver and mediastinal blood pool is an attractive way to minimize variability and to potentially ensure quality of scan from test to retest (Paquet et al., 2004). In our study, however, mean liver SUV max was $11.7 \%$ less in patients with

\section{REFERENCES}

Antoch, G., Vogt, F. M., Freudenberg, L. S., Nazaradeh, F., Goehde, S. C., Barkhausen, J., Dahmen, G., Bockisch, A., Debatin, J. F., and Ruehm, S. G. (2003). Whole-body dualmodality PET/CT and whole-body MRI for tumor staging in oncology. JAMA 290, 3199-3206.

Bar-Shalom, R., Yefremov, N., Guralnik, L., Gaitini, D., Frenkel, A., Kuten, A., Altman, H., Keidar, Z., and Israel, O. (2003). Clinical performance of PET/CT in evaluation of cancer: additional value for diagnostic

infiltration than those without $(2.22 \pm 0.54$ vs. $2.48 \pm 0.6)$ and mean mediastinal SUV max was $9.3 \%$ less in patients with infiltration than those without $(1.72 \pm 0.54$ vs. $1.88 \pm 0.49)$. Therefore, the presence of extravasations may potentially affect not only accuracy but also reproducibility of SUV measurements. In such cases, assessing response to therapy may become more challenging.

The presence of extravasation alone, however, may not be the only factor on affecting SUV measurements. The intensity of the extravasation may have greater affect than the size alone. A visually small site of extravasation with high SUV has more impact on accuracy and reproducibility of SUV measurements than a larger site with a lower SUV. For example, Figure 4 shows extravasation at the edge of the field with SUVmax 1297. Figure 1 shows a visually larger amount of extravasation but a SUVmax of only 196. Although the amount of extravasation visually looks different, the first patient has a high concentration of radioactivity as seen by the intense SUV and has a more profound impact than the second patient.

Our study is not without limitations. The retrospective nature of the study is a potential limitation. Also, the prevalence of extravasation may be underestimated in our study due to the high number of head and neck cases in our institution requiring the arms at the patient's side. Furthermore, the SUV at the site of extravasation was not calculated in all 42 patients. In addition, our study could not adequately assess the impact of the extravasation on the lesion's SUV due to the small sample size. Future studies can better assess the clinical impact of dose extravasation by quantifying the intensity of extravasation and comparing it with the lesion SUV in a larger sample size. Furthermore, the site of injection should be included in clinical practice when possible, but it becomes more important in clinical trials when uptake values are needed for consistency.

\section{CONCLUSION}

We conclude that dose extravasation is commonly encountered $(10.5 \%)$ in PET/CT. However, it is underreported due to omitting injection site from the FOV. When present, extravasations lead to underestimation of SUVmax by an average of $11.7 \%$ in liver and 9.3\% in mediastinum. Extravasations should not only be avoided but also reported in order to avoid false interpretations of the PET/CT exam.

Siegel, B. A. (2010). PET and PET/CT reports: observations from the National Oncologic PET Registry. J. Nucl. Med. 51, 158-163.

Czernin, J., Allen-Auerback, M., and Schelbert, H. R. (2007). Improvements in cancer staging with PET/CT: literature-based evidence as of September 2006. J. Nucl. Med. 48(Suppl. 1), 78S-88S.

Delbeke, D., Coleman, R. E., Guiberteau, M. J., Brown, M. L., Royal, H. D., Siegel, B. A., Townsend, D. W., Berland, L. L., Parker, J. A., Hubner, K., Stabin, M. G., Zubal, G.
Kachelriess, M., Cronin, V., and Holbrook, S. (2006). Procedure guideline for tumor imaging with $18 \mathrm{~F}$ FDG PET/CT 1.0. J. Nucl. Med. 47 885-895.

Graham, M. M., Peterson, L. M., and Hayward, R. M. (2000). Comparison of simplified quantitative analyses of FDG uptake. Nucl. Med. Biol. 27, 647-655.

Hillner, B. E., Siegel, B. A., Shields, A. F., Liu, D., Gareen, I. F., Hunt, E., and Coleman, R. E. (2008). Relationship between cancer type and impact of PET and PET/CT on 
intended management: findings of the national oncologic PET registry. J. Nucl. Med. 49, 1928-1935.

Hilner, B. E., Siegel, B. A., Liu, D., Shields, A. F., Gareen, I. F., Hanna L., Stine, S. H., and Coleman, R. E. (2008). Impact of positron emission tomography/computed tomography and positron emission tomography (PET) alone on expected management of patients with cancer. Initial results from the National Oncologic PET Registry. J Clin. Oncol. 26, 2155-2161.

International Survey of PET/CT Operations and Oncology Imaging. (2010). Academy of Molecular Imaging. Available at: http://www.amiimaging.org/index.php?option $=$ com_content\&task $=$ view\&id $=181$ (accessed August 10, 2011).

Krak, N. C., van der Hoeven, J. J., Hoekstra, O. S., Twisk, J. W., Van der Wall, E., and Lammertsma, A. A. (2003). Measuring [18F]FDG uptake in breast cancer during chemotherapy: comparison of analytical methods.
Eur. J. Nucl. Med. Mol. Imaging 30, 674-681.

Mawlawi, O., and Townsend, D. W. (2009). Multimodality imaging: an update on PET/CT technology. Eur. J. Nucl. Med. Mol. Imaging 36(Suppl. 1), S15-S29.

Osman, M. M., Chaar, B. T., Muzaffar, R., Oliver, D., Reimers, H. J., Walz, B., and Nguyen, N. C. (2010). Added value of true wholebody over limited whole-body $18 \mathrm{~F}-$ FDG PET/CT: initial experience in 500 cancer patients. AJR 195, $1-7$.

Paquet, N., Albert, A., Foidart, J., and Hustinx, R. (2004). Within-patient variability of $18 \mathrm{~F}-\mathrm{FDG}$ : standardized uptake values in normal tissues. J. Nucl. Med. 45, 784-788.

Von Schulthess, G. K., Steinert, H. C., and Hany, T. F. (2006). Integrated PET/CT: current applications and future directions. Radiology 238 , 405-422.

Wahl, R. L., Zasadny, K., Helvie, M., Hutchins, G. D., Weber, B., and Cody, R. (1993). Metabolic monitoring of breast cancer chemohormonotherapy using positron emission tomography: initial evaluation. J. Clin. Oncol. 11 , 2101-2111.

Weber, W. A. (2005). Use of PET for monitoring cancer therapy and for predicting outcome. J. Nucl. Med. 46, 983-995.

Young, H., Baum, R., Cremerius, U., Herholz, K., Hoekstra, O., Lammertsma, A. A., Pruim, J., and Price, P. (1999). Measurement of clinical and subclinical tumor response using [18F]fluorodeoxyglucose and positron emission tomography: review and 1999 EORTC recommendations. European Organization for Research and Treatment of Cancer (EORTC) PET Study Group. Eur. J. Cancer 35 1773-1782.

Conflict of Interest Statement: The authors declare that the research was conducted in the absence of any commercial or financial relationships that could be construed as a potential conflict of interest.

Received: 17 August 2011; paper pending published: 18 September 2011; accepted: 17 October 2011; published online: 16 November 2011.

Citation: Osman MM, Muzaffar R, Altinyay ME and Teymouri C (2011) FDG dose extravasations in PET/CT: frequency and impact on SUV measurements. Front. Oncol. 1:41. doi: 10.3389/fonc.2011.00041

This article was submitted to Frontiers in Cancer Imaging and Diagnosis, a specialty of Frontiers in Oncology.

Copyright (c) 2011 Osman, Muzaffar, Altinyay and Teymouri. This is an openaccess article subject to a non-exclusive license between the authors and Frontiers Media SA, which permits use, distribution and reproduction in other forums, provided the original authors and source are credited and other Frontiers condi tions are complied with. 